\title{
Measurement of the charm production cross section in $\gamma \gamma$ collisions at LEP
}

\section{L3 Collaboration}

M. Acciarri ${ }^{\mathrm{y}}$, P. Achard ${ }^{\mathrm{s}}$, O. Adriani ${ }^{\mathrm{p}}, \mathrm{M}$. Aguilar-Benitez $^{\mathrm{x}}, \mathrm{J}$. Alcaraz ${ }^{\mathrm{x}}$, G. Alemanni ${ }^{\text {u }}$, J. Allaby ${ }^{\mathrm{q}}$, A. Aloisio ${ }^{\text {aa }}$, M.G. Alviggi ${ }^{\text {aa }}$, G. Ambrosi ${ }^{\text {s }}$, H. Anderhub ${ }^{\text {au }}$, V.P. Andreev ${ }^{\text {f,ai }}$, T. Angelescu ${ }^{1}$, F. Anselmo ${ }^{i}$, A. Arefiev ${ }^{z}$, T. Azemoon ${ }^{\text {c }}$, T. Aziz ${ }^{j}$, P. Bagnaia a ${ }^{\text {ah }}$, A. Bajo ${ }^{x}$, L. Baksay ${ }^{\text {ap }}$, A. Balandras ${ }^{d}$, S.V. Baldew ${ }^{b}$, S. Banerjee ${ }^{j}$, Sw. Banerjee ${ }^{\mathrm{d}}$, A. Barczyk ${ }^{\text {au, as }}$, R. Barillère ${ }^{\mathrm{q}}$, P. Bartalini ${ }^{\mathrm{u}}$, M. Basile ${ }^{\mathrm{i}}, \mathrm{N}_{\text {. Batalova }}{ }^{\text {ar }}$,

R. Battiston ${ }^{\text {ae }}$, A. Bay ${ }^{\mathrm{u}}$, F. Becattini ${ }^{\mathrm{p}}, \mathrm{U}$ Becker ${ }^{\mathrm{n}}$, F. Behner ${ }^{\mathrm{au}}$, L. Bellucci $^{\mathrm{p}}$, R. Berbeco ${ }^{c}$, J. Berdugo ${ }^{\mathrm{x}}$, P. Berges ${ }^{\mathrm{n}}$, B. Bertucci ${ }^{\text {ae }}$, B.L. Betev ${ }^{\text {au }}$, S. Bhattacharya ${ }^{\mathrm{j}}$, M. Biasini ${ }^{\text {ae }}$, A. Biland ${ }^{\text {au }}$, J.J. Blaising ${ }^{\text {d }}$, S.C. Blyth ${ }^{\text {af }}$, G.J. Bobbink ${ }^{\text {b }}$, A. Böhm ${ }^{\text {a }}$, L. Boldizsar ${ }^{\mathrm{m}}$, B. Borgia ${ }^{\text {ah }}$, D. Bourilkov ${ }^{\text {au }}$, M. Bourquin ${ }^{\mathrm{s}}, \mathrm{S}$. Braccini ${ }^{\mathrm{s}}$, J.G. Branson ${ }^{\text {am }}$, F. Brochu ${ }^{\mathrm{d}}$, A. Buffini ${ }^{\mathrm{p}}$, A. Buijs ${ }^{\mathrm{aq}}$, J.D. Burger ${ }^{\mathrm{n}}$, W.J. Burger ${ }^{\text {ae }}$, X.D. Cai ${ }^{\mathrm{n}}$, M. Capell ${ }^{\mathrm{n}}$, G. Cara Romeo ${ }^{\mathrm{i}}$, G. Carlino ${ }^{\text {aa }}$, A.M. Cartacci ${ }^{\mathrm{p}}$, J. Casaus ${ }^{\mathrm{x}}$, G. Castellini ${ }^{\mathrm{p}}$, F. Cavallari ${ }^{\text {ah }}$, N. Cavallo ${ }^{\text {aj }}$, C. Cecchi ${ }^{\text {ae }}$, M. Cerrada ${ }^{\mathrm{x}}$, F. Cesaroni ${ }^{\mathrm{v}}$, M. Chamizo ${ }^{\text {, }}$, Y.H. Chang ${ }^{\text {aw }}$, U.K. Chaturvedi ${ }^{\mathrm{r}}$, M. Chemarin ${ }^{\text {w }}$, A. Chen ${ }^{\text {aw }}$, G. Chen ${ }^{\mathrm{g}}$, G.M. Chen ${ }^{\mathrm{g}}$, H.F. Chen ${ }^{\mathrm{t}}$, H.S. Chen ${ }^{\mathrm{g}}$, G. Chiefari ${ }^{\text {aa }}$, L. Cifarelli ${ }^{\mathrm{al}}$, F. Cindolo ${ }^{\mathrm{i}}$,

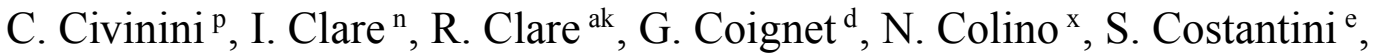
F. Cotorobai ${ }^{1}$, B. de la Cruz $^{\mathrm{x}}$, A. Csilling ${ }^{\mathrm{m}}$, S. Cucciarelli ${ }^{\text {ae }}$, T.S. Dai ${ }^{\mathrm{n}}$, J.A. van Dalen ${ }^{\text {ac }}$, R. D’Alessandro ${ }^{p}$, R. de Asmundis ${ }^{\text {aa }}$, P. Déglon ${ }^{\mathrm{s}}$, A. Degré ${ }^{\mathrm{d}}$, K. Deiters ${ }^{\text {as }}$, D. della Volpe ${ }^{\text {aa }}$, E. Delmeire ${ }^{\text {s }}$, P. Denes ${ }^{\text {ag }}$, F. DeNotaristefani ${ }^{\text {ah }}$, A. De Salvo au, M. Diemoz ${ }^{\text {ah }}$, M. Dierckxsens ${ }^{\text {b }}$, D. van Dierendonck ${ }^{\text {b }}$, C. Dionisi ${ }^{\text {ah }}$, M. Dittmar ${ }^{\text {au }}$, A. Dominguez ${ }^{\text {am }}$, A. Doria ${ }^{\text {aa }}$, M.T. Dova ${ }^{\mathrm{r}, 5}$, D. Duchesneau ${ }^{\mathrm{d}}$, D. Dufournaud ${ }^{\text {d }}$, P. Duinker ${ }^{\text {b }}$, H. El Mamouni ${ }^{\text {w }}$, A. Engler ${ }^{\text {af }}$, F.J. Eppling ${ }^{\text {n }}$, F.C. Erné ${ }^{\text {b }}$, A. Ewers ${ }^{a}$, P. Extermann ${ }^{\text {s }}$, M. Fabre ${ }^{\text {as }}$, M.A. Falagan ${ }^{\mathrm{x}}$, S. Falciano ${ }^{\text {ah, }}$, A. Favara ${ }^{\mathrm{q}}$, J. Fay ${ }^{\text {w }}$, O. Fedin ${ }^{\text {ai }}$, M. Felcini ${ }^{\text {au }}$, T. Ferguson ${ }^{\text {af }}$, H. Fesefeldt ${ }^{\text {a }}$, E. Fiandrini ${ }^{\text {ae }}$, J.H. Field $^{\mathrm{s}}$, F. Filthaut ${ }^{\mathrm{q}}$, P.H. Fisher ${ }^{\mathrm{n}}$, I. Fisk ${ }^{\text {am }}$, G. Forconi ${ }^{\mathrm{n}}$, K. Freudenreich ${ }^{\text {au }}$, C. Furetta ${ }^{\mathrm{y}}$, Yu. Galaktionov ${ }^{\mathrm{z}, \mathrm{n}}$, S.N. Ganguli ${ }^{\mathrm{j}}$, P. Garcia-Abia ${ }^{\mathrm{e}}$, M. Gataullin ${ }^{\text {ad }}$, S.S. Gau ${ }^{\mathrm{k}}$, S. Gentile ${ }^{\text {ah, }}$, N. Gheordanescu ${ }^{1}$, S. Giagu ${ }^{\text {ah }}$, Z.F. Gong ${ }^{\mathrm{t}}$, G. Grenier ${ }^{\mathrm{w}}$, O. Grimm ${ }^{\text {au }}$, M.W. Gruenewald ${ }^{\text {h }}$, M. Guida ${ }^{\text {al }}$, R. van Gulik ${ }^{\text {b }}$, V.K. Gupta ${ }^{\text {ag }}$, A. Gurtu ${ }^{\mathrm{j}}$, L.J. Gutay ${ }^{\text {ar }}$, D. Haas ${ }^{\text {e }}$, A. Hasan ${ }^{\text {ab }}$, D. Hatzifotiadou ${ }^{i}$, T. Hebbeker ${ }^{\text {h }}$, A. Hervé ${ }^{q}$, P. Hidas ${ }^{\mathrm{m}}$, J. Hirschfelder ${ }^{\text {af }}$, H. Hofer ${ }^{\text {au }}$, G. Holzner ${ }^{\text {au }}$, H. Hoorani ${ }^{\text {af }}$, S.R. Hou ${ }^{\text {aw }}$, 
Y. Hu ${ }^{\text {ac }}$, I. Iashvili at, B.N. Jin ${ }^{\mathrm{g}}$, L.W. Jones ${ }^{\text {c }}$, P. de Jong ${ }^{\mathrm{b}}$, I. Josa-Mutuberría ${ }^{\mathrm{x}}$, R.A. Khan ${ }^{\mathrm{r}}$, D. Käfer ${ }^{\mathrm{a}}$, M. Kaur ${ }^{\mathrm{r}, 6}$, M.N. Kienzle-Focacci ${ }^{\text {s }}$, D. Kim ${ }^{\text {ah }}$, J.K. Kim ${ }^{\text {ao }}$, J. Kirkby ${ }^{\mathrm{q}}$, D. Kiss ${ }^{\mathrm{m}}$, W. Kittel ${ }^{\text {ac }}$, A. Klimentov ${ }^{\mathrm{n}, \mathrm{z}}$, A.C. König ${ }^{\mathrm{ac}}$, M. Kopal ${ }^{\text {ar }}$, A. Kopp ${ }^{\text {at }}$, V. Koutsenko ${ }^{\text {n,z }}$, M. Kräber ${ }^{\text {au }}$, R.W. Kraemer ${ }^{\text {af }}$, W. Krenz ${ }^{\text {a }}$, A. Krüger ${ }^{\text {at }}$, A. Kunin ${ }^{\mathrm{n}, \mathrm{z}}$, P. Ladron de Guevara ${ }^{\mathrm{x}}$, I. Laktineh ${ }^{\mathrm{w}}$, G. Landi ${ }^{\mathrm{p}}$, M. Lebeau ${ }^{\mathrm{q}}$, A. Lebedev ${ }^{n}$, P. Lebrun ${ }^{w}$, P. Lecomte ${ }^{\text {au }}$, P. Lecoq ${ }^{\mathrm{q}}$, P. Le Coultre ${ }^{\mathrm{au}}$, H.J. Lee ${ }^{\mathrm{h}}$, J.M. Le Goff ${ }^{\mathrm{q}}$, R. Leiste ${ }^{\text {at }}$, P. Levtchenko ${ }^{\text {ai }}, \mathrm{C}$ Li $^{\mathrm{t}}$, S. Likhoded ${ }^{\text {at }}$, C.H. Lin ${ }^{\text {aw }}$, W.T. Lin ${ }^{\text {aw }}$, F.L. Linde ${ }^{\text {b }}$, L. Lista ${ }^{\text {aa }}$, Z.A. Liu ${ }^{g}$, W. Lohmann ${ }^{\text {at }}$, E. Longo ${ }^{\text {ah }}$, Y.S. Lu ${ }^{\mathrm{g}}$, K. Lübelsmeyer ${ }^{\text {a }}$, C. Luci ${ }^{\text {q,ah }}$, D. Luckey ${ }^{\text {n }}$, L. Lugnier ${ }^{\text {w }}$, L. Luminari ${ }^{\text {ah }}$, W. Lustermann ${ }^{\text {au }}$, W.G. Ma ${ }^{\mathrm{t}}$, M. Maity ${ }^{\mathrm{j}}$, L. Malgeri ${ }^{\mathrm{q}}$, A. Malinin ${ }^{\mathrm{q}}$, C. Maña ${ }^{\mathrm{x}}$, D. Mangeol ${ }^{\text {ac }}$, J. Mans ${ }^{\text {ag }}$, G. Marian ${ }^{\circ}$, J.P. Martin ${ }^{\text {w }}$, F. Marzano ${ }^{\text {ah }}$, K. Mazumdar ${ }^{\mathrm{j}}$, R.R. McNeil ${ }^{\text {f }}$, S. Mele ${ }^{\mathrm{q}}$, L. Merola ${ }^{\text {aa }}$, M. Meschini ${ }^{\text {p }}$, W.J. Metzger ${ }^{\text {ac }}$, M. von der Mey ${ }^{\text {a }}$, A. Mihul ${ }^{1}$, H. Milcent ${ }^{\mathrm{q}}$, G. Mirabelli ${ }^{\text {ah }}$, J. Mnich $^{\mathrm{a}}$, G.B. Mohanty ${ }^{\mathrm{j}}$, T. Moulik ${ }^{\mathrm{j}}$, G.S. Muanza $^{\text {w }}$, A.J.M. Muijs ${ }^{\text {b }}$, B. Musicar ${ }^{\text {am }}$, M. Musy ${ }^{\text {ah }}$, M. Napolitano ${ }^{\text {aa }}$, F. Nessi-Tedaldi ${ }^{\text {au }}$, H. Newman ${ }^{\text {ad }}$, T. Niessen ${ }^{\text {a }}$, A. Nisati ${ }^{\text {ah }}$, H. Nowak ${ }^{\text {at }}$, R. Ofierzynski ${ }^{\text {au }}$, G. Organtini ${ }^{\text {ah }}$, A. Oulianov ${ }^{z}$, C. Palomares ${ }^{\text {x }}$, D. Pandoulas ${ }^{\text {a }}$, S. Paoletti ${ }^{\text {ah,q }}$, P. Paolucci ${ }^{\text {aa }}$, R. Paramatti ${ }^{\text {ah }}$, H.K. Park ${ }^{\text {af }}$, I.H. Park ${ }^{\text {ao }}$, G. Passaleva ${ }^{\text {, }}$, S. Patricelli ${ }^{\text {aa }}$, T. Paul ${ }^{\text {k }}$, M. Pauluzzi ${ }^{\text {ae }}$, C. Paus ${ }^{\text {q }}$, F. Pauss $^{\text {au }}$, M. Pedace ${ }^{\text {ah }}$, S. Pensotti $^{\mathrm{y}}$, D. Perret-Gallix ${ }^{\mathrm{d}}$, B. Petersen ${ }^{\mathrm{ac}}$, D. Piccolo ${ }^{\text {aa }}$, F. Pierella ${ }^{\mathrm{i}}$, M. Pieri ${ }^{\mathrm{p}}$, P.A. Piroué ${ }^{\mathrm{ag}}$, E. Pistolesi ${ }^{\mathrm{y}}$, V. Plyaskin ${ }^{\mathrm{z}}$, M. Pohl ${ }^{\mathrm{s}}$, V. Pojidaev ${ }^{\mathrm{z}, \mathrm{p}}$, H. Postema ${ }^{\mathrm{n}}$, J. Pothier ${ }^{\mathrm{q}}$, D.O. Prokofiev ${ }^{\text {ar }}$, D. Prokofiev ${ }^{\text {ai }}$, J. Quartieri al , G. Rahal-Callot ${ }^{\text {au, }}{ }^{\text {, }}$ M.A. Rahaman ${ }^{j}$,

P. Raics ${ }^{o}$, N. Raja ${ }^{\mathrm{j}}$, R. Ramelli ${ }^{\text {au }}$, P.G. Rancoita ${ }^{\mathrm{y}}$, R. Ranieri $^{\mathrm{p}}$, A. Raspereza ${ }^{\text {at }}$, G. Raven ${ }^{\text {am }}$, P. Razis ${ }^{\text {ab }}$, D. Ren ${ }^{\text {au }}$, M. Rescigno ${ }^{\text {ah }}$, S. Reucroft ${ }^{k}$, S. Riemann ${ }^{\text {at }}$, K. Riles ${ }^{\text {c }}$, J. Rodin ${ }^{\text {ap }}$, B.P. Roe ${ }^{\text {c }}$, L. Romero ${ }^{\mathrm{x}}$, A. Rosca ${ }^{\mathrm{h}}$, S. Rosier-Lees ${ }^{\mathrm{d}}$, S. Roth ${ }^{\text {a }}$, C. Rosenbleck ${ }^{a}$, B. Roux ${ }^{\text {ac }}$, J.A. Rubio ${ }^{q}$, G. Ruggiero ${ }^{\text {p }}$, H. Rykaczewski ${ }^{\text {au }}$, S. Saremi ${ }^{\text {f }}$, S. Sarkar ${ }^{\text {ah }}$ J. Salicio ${ }^{\text {q, E. Sanchez }}{ }^{\text {q, M.P. Sanders }}{ }^{\text {ac }}$, C. Schäfer ${ }^{\text {q }}$, V. Schegelsky ai, S. Schmidt-Kaerst ${ }^{\text {a }}$, D. Schmitz ${ }^{\text {a }}$, H. Schopper ${ }^{\text {av }}$, D.J. Schotanus ${ }^{\text {ac }}$, G. Schwering ${ }^{\text {a }}$, C. Sciacca ${ }^{\text {aa }}$, A. Seganti ${ }^{\text {i }}$, L. Servoli ${ }^{\text {ae }}$, S. Shevchenko ${ }^{\text {ad }}$, N. Shivarov ${ }^{\text {an }}$, V. Shoutko ${ }^{z}$, E. Shumilov ${ }^{z}$, A. Shvorob ${ }^{\text {ad }}$, T. Siedenburg ${ }^{\text {a }}$, D. Son ${ }^{\text {ao }}$, B. Smith ${ }^{\text {af }}$, P. Spillantini ${ }^{\text {p }}$, M. Steuer ${ }^{n}$, D.P. Stickland ${ }^{\text {ag }}$, A. Stone ${ }^{\text {f }}$, B. Stoyanov an , A. Straessner ${ }^{\text {a }}$, K. Sudhakar ${ }^{j}$, G. Sultanov ${ }^{\text {r }}$, L.Z. Sun ${ }^{\mathrm{t}}$, S. Sushkov ${ }^{\text {h }}$, H. Suter ${ }^{\text {au }}$, J.D. Swain ${ }^{\mathrm{r}}$, Z. Szillasi ${ }^{\text {ap, },}$, T. Sztaricskai ${ }^{\text {ap, } 3}$, X.W. Tang ${ }^{\mathrm{g}}$, L. Tauscher ${ }^{\mathrm{e}}$, L. Taylor ${ }^{\mathrm{k}}$, B. Tellili ${ }^{\mathrm{w}}$, D. Teyssier ${ }^{\mathrm{w}}$, C. Timmermans ${ }^{\text {ac }}$, Samuel C.C. Ting ${ }^{n}$, S.M. Ting ${ }^{n}$, S.C. Tonwar ${ }^{\mathrm{j}}$, J. Tóth ${ }^{\mathrm{m}}$, C. Tully ${ }^{\mathrm{q}}$, K.L. Tung ${ }^{\mathrm{g}}$, Y. Uchida ${ }^{\mathrm{n}}$, J. Ulbricht ${ }^{\text {au }}$, E. Valente ${ }^{\text {ah }}$, G. Vesztergombi ${ }^{\mathrm{m}}$, I. Vetlitsky ${ }^{\mathrm{z}}$, D. Vicinanza ${ }^{\text {al }}$, G. Viertel ${ }^{\text {au }}$, S. Villa ${ }^{k}$, M. Vivargent ${ }^{\mathrm{d}}, \mathrm{S}$. Vlachos ${ }^{\mathrm{e}}$, I. Vodopianov ${ }^{\text {ai }}$, H. Vogel ${ }^{\text {af }}, \mathrm{H}$. Vogt ${ }^{\text {at }}$, I. Vorobiev ${ }^{\text {af }}$, A.A. Vorobyov ${ }^{\text {ai }}$, A. Vorvolakos ${ }^{\text {ab }}$, M. Wadhwa ${ }^{\text {e, }}$, W. Wallraff ${ }^{\mathrm{a}}$, M. Wang ${ }^{\mathrm{n}}$, X.L. Wang ${ }^{\mathrm{t}}$, Z.M. Wang ${ }^{\mathrm{t}}$, A. Weber ${ }^{\mathrm{a}}$, M. Weber ${ }^{\mathrm{a}}$, P. Wienemann ${ }^{\text {a }}$, H. Wilkens ${ }^{\text {ac }}$, S.X. Wu ${ }^{\text {n }}$, S. Wynhoff ${ }^{\mathrm{q}}$, L. Xia ${ }^{\text {ad }}$, Z.Z. Xu ${ }^{\mathrm{t}}$, 
J. Yamamoto ${ }^{\text {c }}$, B.Z. Yang ${ }^{\mathrm{t}}$, C.G. Yang ${ }^{\mathrm{g}}$, H.J. Yang ${ }^{\mathrm{g}}$, M. Yang ${ }^{\mathrm{g}}$, J.B. Ye ${ }^{\mathrm{t}}$, S.C. Yeh ${ }^{\mathrm{ax}}$, An. Zalite ${ }^{\text {ai }}$, Yu. Zalite ${ }^{\text {ai }}$, Z.P. Zhang ${ }^{\mathrm{t}}$, G.Y. Zhu ${ }^{\mathrm{g}}$, R.Y. Zhu ${ }^{\text {ad }}$, A. Zichichi ${ }^{\mathrm{i}, \mathrm{q}, \mathrm{r}}$, G. Zilizi ${ }^{\text {ap,3 }}$, B. Zimmermann ${ }^{\text {au }}$, M. Zöller ${ }^{\text {a }}$

\author{
a I. Physikalisches Institut, RWTH, D-52056 Aachen, Germany \\ and III. Physikalisches Institut, RWTH, D-52056 Aachen, Germany ${ }^{1}$ \\ b National Institute for High Energy Physics, NIKHEF, \\ and University of Amsterdam, NL-1009 DB Amsterdam, The Netherlands \\ ${ }^{\mathrm{c}}$ University of Michigan, Ann Arbor, MI 48109, USA \\ d Laboratoire d'Annecy-le-Vieux de Physique des Particules, LAPP, IN2P3-CNRS, BP 110, F-74941 Annecy-le-Vieux Cedex, France \\ ${ }^{\mathrm{e}}$ Institute of Physics, University of Basel, CH-4056 Basel, Switzerland \\ ${ }^{\mathrm{f}}$ Louisiana State University, Baton Rouge, LA 70803, USA \\ $\mathrm{g}$ Institute of High Energy Physics, IHEP, 100039 Beijing, China ${ }^{7}$ \\ ${ }^{\mathrm{h}}$ Humboldt University, D-10099 Berlin, Germany ${ }^{1}$ \\ ${ }^{\mathrm{i}}$ University of Bologna and INFN-Sezione di Bologna, I-40126 Bologna, Italy \\ $\mathrm{j}$ Tata Institute of Fundamental Research, Bombay 400005 , India \\ ${ }^{\mathrm{k}}$ Northeastern University, Boston, MA 02115, USA \\ ${ }^{1}$ Institute of Atomic Physics and University of Bucharest, R-76900 Bucharest, Romania \\ $\mathrm{m}$ Central Research Institute for Physics of the Hungarian Academy of Sciences, H-1525 Budapest 114, Hungary ${ }^{2}$ \\ ${ }^{\mathrm{n}}$ Massachusetts Institute of Technology, Cambridge, MA 02139, USA \\ ${ }^{\circ}$ KLTE-ATOMKI, H-4010 Debrecen, Hungary ${ }^{3}$ \\ $\mathrm{p}$ INFN-Sezione di Firenze and University of Florence, I-50125 Florence, Italy \\ ${ }^{\mathrm{q}}$ European Laboratory for Particle Physics, CERN, CH-1211 Geneva 23, Switzerland \\ ${ }^{\mathrm{r}}$ World Laboratory, FBLJA Project, CH-1211 Geneva 23, Switzerland \\ ${ }^{\mathrm{s}}$ University of Geneva, CH-1211 Geneva 4, Switzerland \\ ${ }^{\mathrm{t}}$ Chinese University of Science and Technology, USTC, Hefei, Anhui 230 029, China ${ }^{7}$ \\ " University of Lausanne, CH-1015 Lausanne, Switzerland \\ ${ }^{v}$ INFN-Sezione di Lecce and Università Degli Studi di Lecce, I-73100 Lecce, Italy \\ ${ }^{\mathrm{w}}$ Institut de Physique Nucléaire de Lyon, IN2P3-CNRS, Université Claude Bernard, F-69622 Villeurbanne, France \\ ${ }^{\mathrm{x}}$ Centro de Investigaciones Energéticas, Medioambientales y Tecnologícas, CIEMAT, E-28040 Madrid, Spain ${ }^{4}$ \\ y INFN-Sezione di Milano, I-20133 Milan, Italy \\ ${ }^{\mathrm{z}}$ Institute of Theoretical and Experimental Physics, ITEP, Moscow, Russia \\ aa INFN-Sezione di Napoli and University of Naples, I-80125 Naples, Italy \\ ${ }^{\mathrm{ab}}$ Department of Natural Sciences, University of Cyprus, Nicosia, Cyprus \\ ac University of Nijmegen and NIKHEF, NL-6525 ED Nijmegen, The Netherlands \\ ad California Institute of Technology, Pasadena, CA 91125, USA \\ ae INFN-Sezione di Perugia and Università Degli Studi di Perugia, I-06100 Perugia, Italy \\ af Carnegie Mellon University, Pittsburgh, PA 15213, USA \\ ag Princeton University, Princeton, NJ 08544, USA \\ ah INFN-Sezione di Roma and University of Rome, "La Sapienza", I-00185 Rome, Italy \\ ai Nuclear Physics Institute, St. Petersburg, Russia \\ aj INFN-Sezione di Napoli and University of Potenza, I-85100 Potenza, Italy \\ ${ }^{\text {ak }}$ University of California, Riverside, CA 92521, USA \\ al University and INFN, Salerno, I-84100 Salerno, Italy \\ am University of California, San Diego, CA 92093, USA \\ an Bulgarian Academy of Sciences, Central Lab. of Mechatronics and Instrumentation, BU-1113 Sofia, Bulgaria \\ ${ }^{\text {ao }}$ Laboratory of High Energy Physics, Kyungpook National University, 702-701 Taegu, South Korea \\ ap University of Alabama, Tuscaloosa, AL 35486, USA \\ aq Utrecht University and NIKHEF, NL-3584 CB Utrecht, The Netherlands \\ ${ }^{a r}$ Purdue University, West Lafayette, IN 47907, USA \\ as Paul Scherrer Institut, PSI, CH-5232 Villigen, Switzerland \\ at DESY, D-15738 Zeuthen, Germany

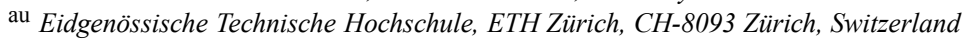 \\ av University of Hamburg, D-22761 Hamburg, Germany \\ aw National Central University, Chung-Li, Taiwan, ROC \\ ${ }^{a x}$ Department of Physics, National Tsing Hua University, Taiwan, ROC
}




\begin{abstract}
Open charm production in $\gamma \gamma$ collisions is studied with data collected at $\mathrm{e}^{+} \mathrm{e}^{-}$centre-of-mass energies from $189 \mathrm{GeV}$ to $202 \mathrm{GeV}$ corresponding to a total integrated luminosity of $410 \mathrm{pb}^{-1}$. The charm cross section $\sigma(\gamma \gamma \rightarrow \mathrm{c} \overline{\mathrm{c} X})$ is measured for the first time as a function of the two-photon centre-of-mass energy in the interval from $5 \mathrm{GeV}$ to $70 \mathrm{GeV}$ and is compared to NLO QCD calculations. ๑ 2001 Published by Elsevier Science B.V.
\end{abstract}

\section{Introduction}

The cross section for the interaction of two quasireal photons is described by three components. The first "soft" component is described by the Vector Dominance Model (VDM), parametrized in hadronic phenomenology by Regge poles. A second "direct" component, the point like reaction $\gamma \gamma \rightarrow \mathrm{q} \overline{\mathrm{q}}$, can be calculated in QED. Finally, there is a "hard component" (resolved or anomalous QCD component) which requires knowledge of the quark and gluon parton density functions of the photon. The direct and hard contributions to two-photon interactions can be measured in open heavy flavour production. In this case a hard physical scale is given by the c- or b-quark mass. At LEP energies, the direct and single resolved processes, shown in Fig. 1, are predicted to give comparable contributions to the charm production cross section [1], whereas at low energies the direct process dominates. The main contribution to the resolved photon cross section is the photon-gluon fusion process $\gamma \mathrm{g} \rightarrow \mathrm{c} \overline{\mathrm{c}}$. Contributions to charm production arising from VDM

\footnotetext{
1 Supported by the German Bundesministerium für Bildung, Wissenschaft, Forschung und Technologie.

2 Supported by the Hungarian OTKA fund under contract numbers T019181, F023259 and T024011.

3 Also supported by the Hungarian OTKA fund under contract numbers T22238 and T026178.

4 Supported also by the Comisión Interministerial de Ciencia y Tecnología.

5 Also supported by CONICET and Universidad Nacional de La Plata, CC 67, 1900 La Plata, Argentina.

6 Also supported by Panjab University, Chandigarh-160014, India.

7 Supported by the National Natural Science Foundation of China.
}

processes and from doubly resolved processes are expected to be small [1].

This Letter presents the measurement of the $\gamma \gamma \rightarrow$ c $\bar{c} X$ cross section as a function of the two-photon centre-of-mass energy $W_{\gamma \gamma}$ in the interval $5 \mathrm{GeV} \leqslant$ $W_{\gamma \gamma} \leqslant 70 \mathrm{GeV}$. The data correspond to a total integrated luminosity $\mathcal{L}=410 \mathrm{pb}^{-1}$, collected with the L3 detector [2] at centre-of-mass energies $\sqrt{\mathrm{s}}=$ 189-202 GeV. The inclusive charm production cross section $\sigma\left(\mathrm{e}^{+} \mathrm{e}^{-} \rightarrow \mathrm{e}^{+} \mathrm{e}^{-} \mathrm{c} \overline{\mathrm{c} X}\right)$ was measured by $\mathrm{L} 3$ at $\sqrt{\mathrm{s}}=91-202 \mathrm{GeV}[3,4]$. Charm quarks are identified by their decay into electrons [4]. ${ }^{8}$

\section{Monte Carlo}

The PYTHIA [5] Monte Carlo is used to model the $\mathrm{e}^{+} \mathrm{e}^{-} \rightarrow \mathrm{e}^{+} \mathrm{e}^{-}$hadrons processes. Quarks other than $\mathrm{b}$ are taken as massless in the corresponding matrix elements [6]. The resolved process uses the SaS1d photon structure function [7]. The two-photon luminosity function is implemented in the equivalent photon approximation (EPA) [8] with a cutoff $Q^{2}<$ $m_{\rho}^{2}$, where $m_{\rho}$ is the mass of the $\rho$ meson.

The background sources are $\mathrm{e}^{+} \mathrm{e}^{-} \rightarrow \mathrm{e}^{+} \mathrm{e}^{-} \tau^{+} \tau^{-}$, $\mathrm{e}^{+} \mathrm{e}^{-} \rightarrow \mathrm{q} \overline{\mathrm{q}}, \mathrm{e}^{+} \mathrm{e}^{-} \rightarrow \tau^{+} \tau^{-}$and $\mathrm{e}^{+} \mathrm{e}^{-} \rightarrow \mathrm{W}^{+} \mathrm{W}^{-}$. These processes are generated by JAMVG [9], PYTHIA, KORALZ [10] and KORALW [11], respectively. The detector simulation is performed using the GEANT [12] and GHEISHA [13] packages. The Monte Carlo events are reconstructed in the same way as the data. Time dependent detector inefficiencies, as monitored during the data taking period, are also simulated.

\footnotetext{
8 Electron stands for electron or positron throughout this Letter.
} 


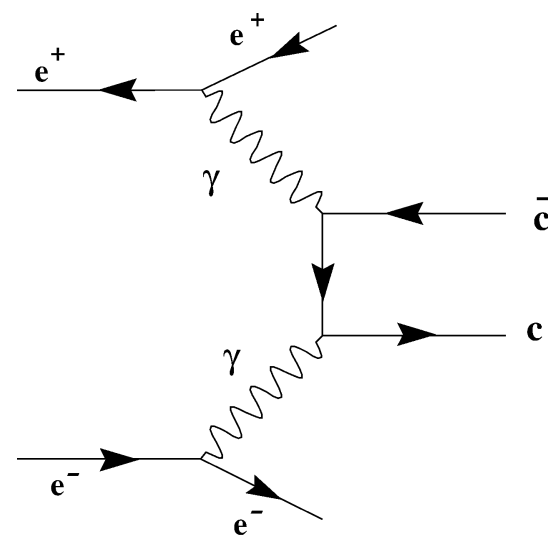

Direct

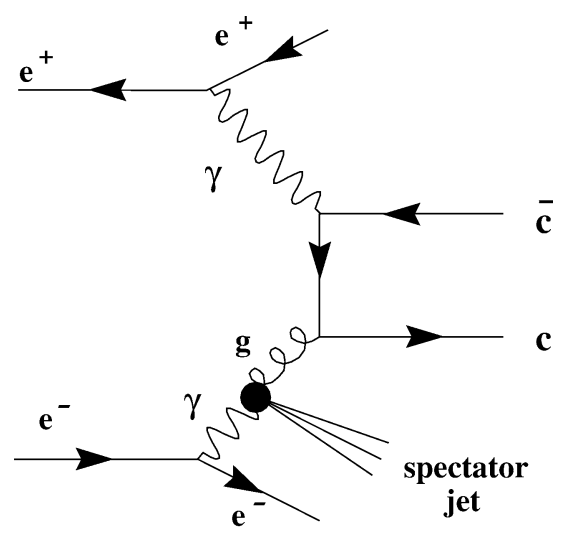

Single Resolved

Fig. 1. Diagrams contributing to charm production in $\gamma \gamma$ collisions.

\section{Measurement of $\sigma(\gamma \gamma \rightarrow \mathrm{c} \overline{\mathbf{c} X})$}

The measurement of the $\sigma(\gamma \gamma \rightarrow \mathrm{c} \overline{\mathrm{c} X})$ cross section is performed on the high statistics electron sample of 2434 events discussed in Ref. [4]. The backgrounds from annihilation processes and two-photon production of tau pairs are estimated to be $0.75 \%$ and are subtracted from the data. The background from $\gamma \gamma \rightarrow b \bar{b} X$ events is modelled with the PYTHIA Monte Carlo assuming equal contributions from direct and resolved processes. It is also subtracted from the data assuming our measured cross section [4], $13.1 \pm 2.0$ (stat.) \pm 2.4 (syst.) pb. The charm purity after background subtraction is $75 \%$ and the measured $\mathrm{e}^{+} \mathrm{e}^{-} \rightarrow \mathrm{e}^{+} \mathrm{e}^{-} \mathrm{c} \overline{\mathrm{c} X}$ cross section is [4]: $1072 \pm$ 33 (stat) \pm 126 (syst) pb.

The analysis is restricted to events with visible mass $W_{\text {vis }}>3 \mathrm{GeV}$. $W_{\text {vis }}$ is calculated from the fourmomentum vectors of the measured particles, tracks and calorimetric clusters including those from the small angle luminosity monitor. The $W_{\text {vis }}$ distribution is corrected for trigger efficiency using data with a set of independent triggers. It varies from $94 \%$ at $W_{\text {vis }}=$ $3 \mathrm{GeV}$ to $98 \%$ at $W_{\text {vis }} \geqslant 30 \mathrm{GeV}$. Table 1 shows the selected data events in bins of $W_{\text {vis }}$ and the different contributions of the signal and the background predicted by the PYTHIA Monte Carlo. A comparison of the visible mass distribution of the data after final
Table 1

The number of events for the data, $N_{\text {data }}$, the expected signal, $N_{\mathrm{c} \overline{\mathrm{c}}}$, the background from light flavours, $N_{\mathrm{uds}}$, b production $N_{\mathrm{b}}$ and other processes $N_{\mathrm{bkgd}}$ as a function of $W_{\mathrm{vis}}$

\begin{tabular}{crrrrc}
\hline$W_{\text {vis }}(\mathrm{GeV})$ & $N_{\text {data }}$ & $N_{\mathrm{c} \overline{\mathrm{c}}}$ & $N_{\text {uds }}$ & $N_{\mathrm{b}}$ & $N_{\text {bkgd }}$ \\
\hline $3-5$ & 261 & 208 & 48 & 0.2 & 4.6 \\
$5-10$ & 741 & 519 & 186 & 31 & 5.3 \\
$10-15$ & 527 & 360 & 116 & 50 & 1.3 \\
$15-25$ & 541 & 352 & 127 & 61 & 1.1 \\
$25-40$ & 276 & 183 & 55 & 36 & 2.3 \\
$40-70$ & 85 & 47 & 22 & 12 & 3.8 \\
$>70$ & 3 & 0.3 & 2 & 0.5 & 0.2 \\
\hline
\end{tabular}

selection with the expectations of the PYTHIA Monte Carlo at $\sqrt{s}=189-202 \mathrm{GeV}$ is shown in Fig. 2.

\subsection{Unfolding procedure}

The hadronic final state is not always fully contained in the detector acceptance. An unfolding procedure is hence applied to obtain the $\gamma \gamma$ centre-of-mass energy $W_{\gamma \gamma}$ from $W_{\text {vis }}$. Fig. 3 shows the correlation of the $W_{\text {vis }}$ average value, $\left\langle W_{\text {vis }}\right\rangle$, with $W_{\gamma \gamma}$ as predicted by the Monte Carlo. The unfolding corrects for both missing particles and detector resolution by the 


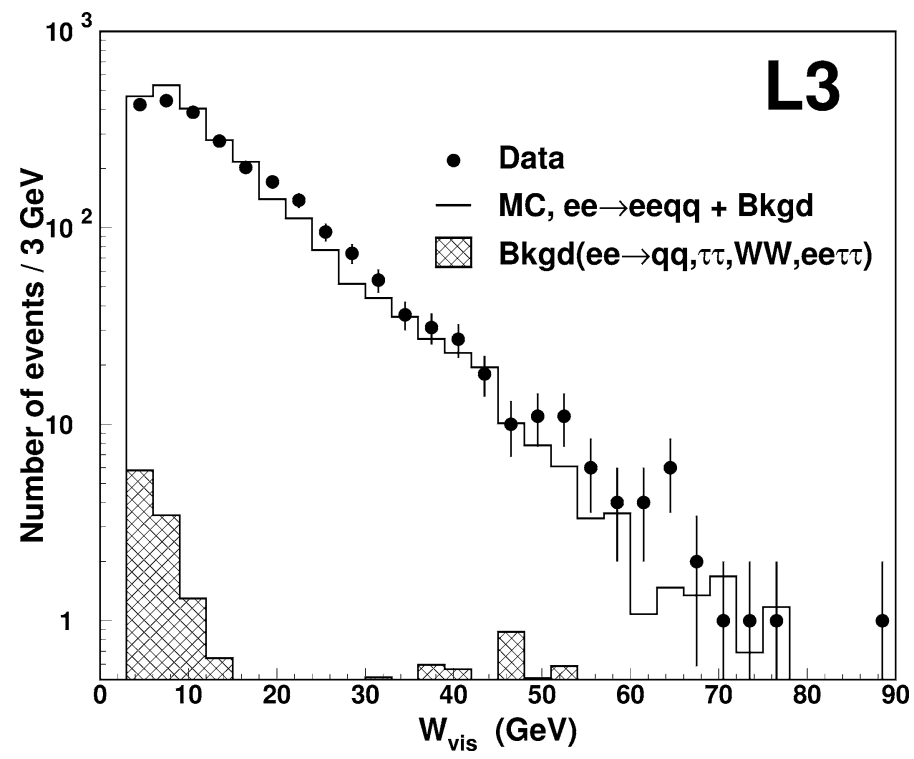

Fig. 2. The visible mass spectrum, $W_{\text {vis }}$, for the inclusive electron data at $\sqrt{s}=189-202 \mathrm{GeV}$ compared to the PYTHIA prediction. The Monte Carlo spectrum with all flavour contributions is normalized to the integrated luminosity of the data after scaling to the measured charm and beauty cross sections according to our measurements [4]. The fraction of uds events is scaled according to the estimate of the charm purity of the selected sample [4]. Other background sources are also shown.

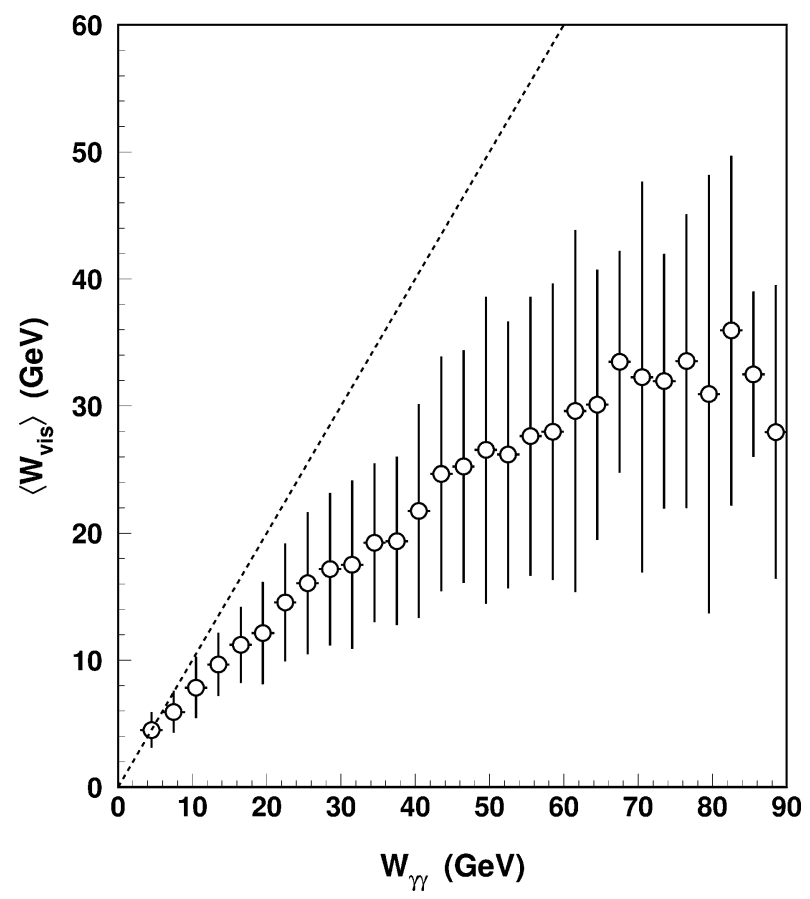

Fig. 3. Profile of the distribution of $W_{\text {vis }}$ as a function of $W_{\gamma \gamma}$ using the PYTHIA Monte Carlo. The open circles correspond to the mean values and the error bars represent the r.m.s. of the distribution. The dashed line corresponds to perfect correlation. 
Table 2

Unfolded number of charm events, $N_{\mathrm{c} \overline{\mathrm{c}}}$, efficiencies and cross section values as a function of $W_{\gamma \gamma}$. The first uncertainty on the cross section is statistical and the second is systematic. The cross section values are evaluated at the centre of each $W_{\gamma \gamma}$ interval

\begin{tabular}{|c|c|c|c|c|}
\hline$\Delta W_{\gamma \gamma}(\mathrm{GeV})$ & $N_{\mathrm{c} \overline{\mathrm{c}}}$ unfolded & Efficiency (\%) & $\Delta \sigma\left(\mathrm{e}^{+} \mathrm{e}^{-} \rightarrow \mathrm{e}^{+} \mathrm{e}^{-} \mathrm{c} \overline{\mathrm{c} X}\right)(\mathrm{nb})$ & $\sigma(\gamma \gamma \rightarrow \mathrm{c} \overline{\mathrm{c} X})(\mathrm{nb})$ \\
\hline $5-10$ & 428 & $0.33 \pm 0.01$ & $0.316 \pm 0.013 \pm 0.040$ & $21.0 \pm 0.8 \pm 2.9$ \\
\hline $10-15$ & 268 & $0.56 \pm 0.02$ & $0.117 \pm 0.004 \pm 0.015$ & $18.6 \pm 0.7 \pm 2.6$ \\
\hline $15-25$ & 389 & $0.70 \pm 0.02$ & $0.136 \pm 0.005 \pm 0.018$ & $24.2 \pm 0.9 \pm 3.4$ \\
\hline $25-40$ & 325 & $0.72 \pm 0.03$ & $0.110 \pm 0.004 \pm 0.015$ & $33.0 \pm 1.3 \pm 4.9$ \\
\hline $40-70$ & 196 & $0.57 \pm 0.03$ & $0.084 \pm 0.004 \pm 0.013$ & $38.8 \pm 1.9 \pm 6.2$ \\
\hline
\end{tabular}

relation:

$N\left(W_{\gamma \gamma}(i)\right)=\sum_{j} A_{i j} N\left(W_{\mathrm{vis}}(j)\right)$

where $N\left(W_{\gamma \gamma}(i)\right)$ is the content of the $i$ th interval of $W_{\gamma \gamma}$ listed in Table 2, and $N\left(W_{\text {vis }}(j)\right)$ is the content of the $j$ th interval of $W_{\text {vis }}$ listed in Table 1 . The matrix $A_{i j}$ is constructed by considering for each Monte Carlo event its reconstructed $W_{\text {vis }}$ and generated $W_{\gamma \gamma}[14]$ :

$A_{i j}=\frac{P\left(W_{\mathrm{vis}}(j) \mid W_{\gamma \gamma}(i)\right) P\left(W_{\gamma \gamma}(i)\right)}{\sum_{l} P\left(W_{\mathrm{vis}}(j) \mid W_{\gamma \gamma}(l)\right) P\left(W_{\gamma \gamma}(l)\right)}$,

where $P\left(W_{\text {vis }} \mid W_{\gamma \gamma}\right)$ is the likelihood of observing the measured value $W_{\text {vis }}$ given a generated value of $W_{\gamma \gamma}$ and $P\left(W_{\gamma \gamma}\right)$ is the generated $W_{\gamma \gamma}$ distribution after the selection cuts.

The unfolding matrix is determined using charm events generated with PYTHIA. The unfolding uncertainty is estimated with PYTHIA using five flavour events instead of charm events and using the PHOJET [15] Monte Carlo generator with all quark flavours. This comparison leads to an estimated uncertainty of $5 \%$. Charm production in the PHOJET generator is implemented with a high $p_{t}$ threshold which prevents the use of PHOJET for charm efficiency studies.

After unfolding, the events are corrected for efficiency using the ratio between selected and generated charm events in each $W_{\gamma \gamma}$ interval. The events with $3 \mathrm{GeV} \leqslant W_{\text {vis }} \leqslant 5 \mathrm{GeV}$ and $W_{\text {vis }} \geqslant 70 \mathrm{GeV}$ are used for unfolding, but are excluded from the measurement due to their large correction factors and unfolding uncertainty.

\subsection{Cross section measurement}

The cross section $\Delta \sigma\left(\mathrm{e}^{+} \mathrm{e}^{-} \rightarrow \mathrm{e}^{+} \mathrm{e}^{-} \mathrm{c} \overline{\mathrm{c} X}\right)$ is measured from the number of events corrected for the efficiency with the PYTHIA Monte Carlo in each $W_{\gamma \gamma}$ interval and the integrated luminosity.

To extract the cross section $\sigma(\gamma \gamma \rightarrow \operatorname{cc} \mathrm{X})$ of two real photons, the photon flux $\mathcal{L}_{\gamma \gamma}$ [8] is calculated and the hadronic two-photon process is extrapolated to $Q^{2}=0$, where $Q^{2}$ is the virtuality of a photon. This is done through the following relation:

$$
\begin{aligned}
& \Delta \sigma\left(\mathrm{e}^{+} \mathrm{e}^{-} \rightarrow \mathrm{e}^{+} \mathrm{e}^{-} \mathrm{c} \overline{\mathrm{c} X}\right) \\
& =\int \frac{d Q_{1}^{2}}{Q_{1}^{2}} \frac{d Q_{2}^{2}}{Q_{2}^{2}} \mathcal{L}_{\gamma \gamma}\left(W_{\gamma \gamma}, Q_{1}^{2}, Q_{2}^{2}\right) \\
& \quad \times \sigma_{\gamma \gamma \rightarrow \mathrm{c} \overline{\mathrm{C}}}\left(W_{\gamma \gamma}\right) d W_{\gamma \gamma} .
\end{aligned}
$$

For each $W_{\gamma \gamma}$ interval a numerical integration is performed over its width and the unmeasured $Q^{2}$ of the scattered electrons.

Table 2 gives the efficiencies and the cross sections $\Delta \sigma\left(\mathrm{e}^{+} \mathrm{e}^{-} \rightarrow \mathrm{e}^{+} \mathrm{e}^{-} \operatorname{c\overline {c}X}\right)$ and $\sigma(\gamma \gamma \rightarrow \mathrm{c} \overline{\mathrm{c} X})$ as a function of $W_{\gamma \gamma}$. The systematic uncertainties arise mainly from the charm purity estimate, followed by the unfolding procedure, the charm efficiency, the beauty cross section [4], the photon flux and the trigger efficiency, all summarized in Table 3. The photon flux uncertainty is estimated by the comparison of the two models $[8,16]$. The uncertainty due to the other background processes is negligible. Due to the unfolding, the data points are correlated. The correlation matrix is given in Table 4 . 
Table 3

Systematic uncertainties (in percent) on the cross section for the process $\gamma \gamma \rightarrow \operatorname{cc} X$

\begin{tabular}{|c|c|c|c|c|c|}
\hline \multirow[t]{2}{*}{ Source of uncertainty } & \multicolumn{5}{|c|}{$W_{\gamma \gamma}$} \\
\hline & $5-10 \mathrm{GeV}$ & $10-15 \mathrm{GeV}$ & $15-25 \mathrm{GeV}$ & $25-40 \mathrm{GeV}$ & $40-70 \mathrm{GeV}$ \\
\hline Charm purity & 11.3 & 12.2 & 12.2 & 12.6 & 12.6 \\
\hline Unfolding & 5.0 & 5.0 & 5.0 & 5.0 & 5.0 \\
\hline Charm efficiency & 3.8 & 3.7 & 3.3 & 3.9 & 5.0 \\
\hline$\sigma\left(\mathrm{e}^{+} \mathrm{e}^{-} \rightarrow \mathrm{e}^{+} \mathrm{e}^{-} \mathrm{b} \overline{\mathrm{b}} \mathrm{X}\right)$ & 3.5 & 1.6 & 2.8 & 3.4 & 4.1 \\
\hline Photon flux & 0.1 & 1.0 & 1.5 & 2.7 & 4.8 \\
\hline Trigger efficiency & 2.1 & 2.1 & 2.2 & 2.3 & 2.3 \\
\hline Total & 13.6 & 14.0 & 14.1 & 14.9 & 15.9 \\
\hline
\end{tabular}

Table 4

Correlation matrix of the data after unfolding

\begin{tabular}{clllll}
\hline$W_{\gamma \gamma}(\mathrm{GeV})$ & $5-10$ & $10-15$ & $15-25$ & $25-40$ & $40-70$ \\
\hline $5-10$ & 1.0 & & & & \\
$10-15$ & 0.739 & 1.0 & & & \\
$15-25$ & 0.294 & 0.703 & 1.0 & & \\
$25-40$ & 0.090 & 0.302 & 0.757 & 1.0 & \\
$40-70$ & 0.014 & 0.093 & 0.388 & 0.778 & 1.0 \\
\hline
\end{tabular}

\section{Comparison with theory and interpretation}

Fig. 4 shows the differential cross section $\Delta \sigma\left(\mathrm{e}^{+} \mathrm{e}^{-}\right.$ $\left.\rightarrow \mathrm{e}^{+} \mathrm{e}^{-} \mathrm{c} \overline{\mathrm{c} X}\right) / \Delta W_{\gamma \gamma}$ as a function of $W_{\gamma \gamma}$ measured in the interval $5 \mathrm{GeV} \leqslant W_{\gamma \gamma} \leqslant 70 \mathrm{GeV}$. The expected slope from the PYTHIA Monte Carlo is steeper than that of the data.

Fig. 5 compares the measured cross section $\sigma(\gamma \gamma \rightarrow$ cc $\mathrm{X})$ as a function of $W_{\gamma \gamma}$ with NLO QCD calculations [17]. The calculations use massive quarks in the matrix elements. The charm mass, $m_{\mathrm{c}}$, is fixed to 1.2 GeV, the renormalization and factorization scales are set to $m_{\mathrm{c}}$ and $2 m_{\mathrm{c}}$, respectively, the QCD parameter $\Lambda_{5}^{\mathrm{QCD}}$ is set at $227.5 \mathrm{MeV}$, and the GRS-HO [18] photon parton density function is used. Using this set of input parameters, the NLO QCD predictions reproduce well the energy dependence and the normalization. The calculation with $m_{\mathrm{c}}=1.5 \mathrm{GeV}$ results in about $50 \%$ lower cross section values, except the first point, where it is lower by $25 \%$. A change in the renor- malization scale from $m_{\mathrm{c}}$ to $2 m_{\mathrm{c}}$ decreases the QCD prediction by $10 \%$ and $30 \%$ at low and high $W_{\gamma \gamma}$, respectively.

We compare also the measured charm cross section with the total cross section of hadron production in two-photon collisions [19], scaled by an arbitrary factor $1 / 20$. The slope of $\sigma(\gamma \gamma \rightarrow \mathrm{c} \overline{\mathrm{C} X})$ is clearly larger than that of $\sigma(\gamma \gamma \rightarrow$ hadrons $)$.

A more quantitative comparison results from fits to the data. A parametrisation [20] of the form $\sigma=$ $A s^{\epsilon}+B s^{-\eta}$, "Pomeron + Reggeon", with $s=W_{\gamma \gamma}^{2}$ describes well the energy behaviour of all the total hadron-hadron cross sections with universal values $\epsilon=0.093 \pm 0.002$ and $\eta=0.358 \pm 0.015$ [16]. A fit to our data with $\epsilon, A$ and $B$ being free parameters and with fixed $\eta=0.358$ yields in the interval $5 \mathrm{GeV} \leqslant$ $W_{\gamma \gamma} \leqslant 70 \mathrm{GeV}$ :

$\epsilon=0.40 \pm 0.03$ (stat.) \pm 0.07 (syst.),

$A=1.3 \pm 0.3$ (stat.) \pm 0.7 (syst.) nb,

$B=44.0 \pm 3.8$ (stat.) \pm 11.1 (syst.) nb,

$\chi^{2} /$ d.o.f. $=8.9 / 2$.

Correlations between the data points are taken into account. The systematic uncertainty on each parameter is estimated by performing fits with total uncertainties and statistical uncertainties only in $\chi^{2}$. The systematic uncertainties are then evaluated by subtracting in quadrature the uncertainty of the statistical only fit from that given by the total uncertainties. The fitted value of $\epsilon$ is higher than the universal value [16]. 


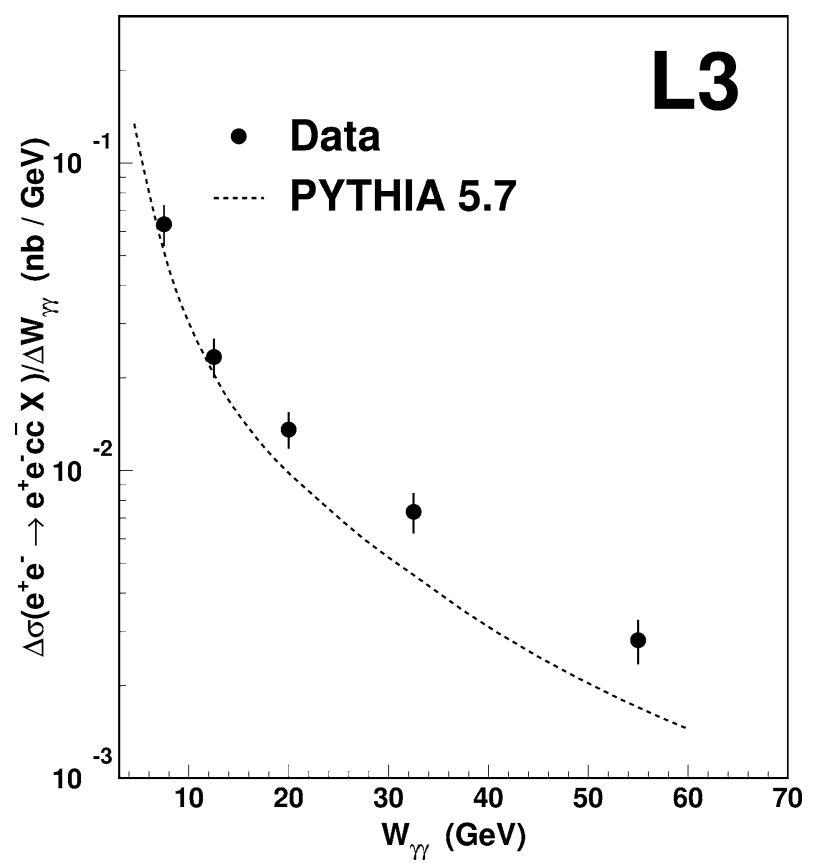

Fig. 4. The cross section $\Delta \sigma\left(\mathrm{e}^{+} \mathrm{e}^{-} \rightarrow \mathrm{e}^{+} \mathrm{e}^{-} \mathrm{c} \overline{\mathrm{cX}}\right) / \Delta W_{\gamma \gamma}$ as a function of $W_{\gamma \gamma}$ at $\sqrt{s}=189-202 \mathrm{GeV}$. The dashed line corresponds to the leading order PYTHIA Monte Carlo prediction.

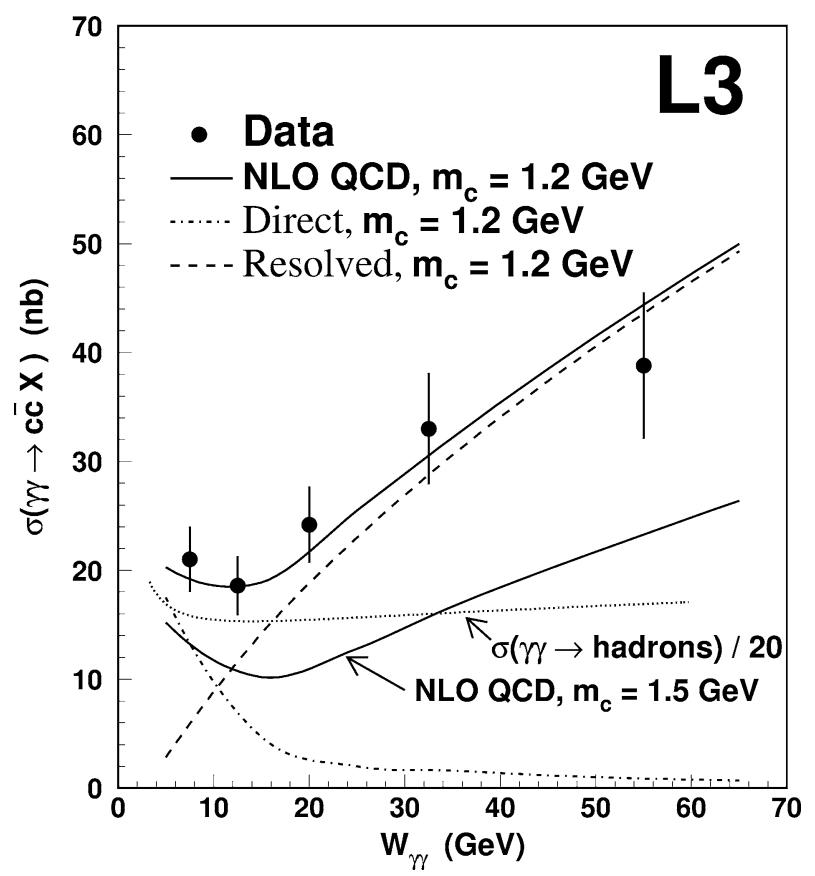

Fig. 5. The cross section $\sigma(\gamma \gamma \rightarrow \mathrm{c} \overline{\mathrm{c} X})$ as a function of $W_{\gamma \gamma}$ at $\sqrt{s}=189-202 \mathrm{GeV}$. The dotted curve is the total cross section $\sigma(\gamma \gamma \rightarrow$ hadrons $)$ measured by L3 [19] scaled by an arbitrary factor $1 / 20$. The continuous line is the NLO QCD prediction, while the dashed-dotted and dashed curves show the expectation from the direct and resolved process, respectively. 
Modifications to the parametrization of the total cross section were recently proposed [21]. In order to allow for a larger slope for the high energy behaviour, an admixture of "hard" and "soft" Pomerons with the Pomeron powers $\epsilon$ of 0.4 and 0.1 , respectively, was introduced. Our data correspond to a direct measurement of the "hard" component of photonphoton collisions.

A fit to the data by the form $\sigma=A s^{\epsilon}$, performed in the interval $10 \mathrm{GeV} \leqslant W_{\gamma \gamma} \leqslant 70 \mathrm{GeV}$, yields:

$\epsilon=0.26 \pm 0.02$ (stat.) \pm 0.03 (syst.),

$A=5.0 \pm 0.4$ (stat.) \pm 0.8 (syst.) nb,

$\chi^{2} /$ d.o.f. $=1.9 / 2$.

The value of $\epsilon$ is in agreement with a similar fit to the measurements of the photoproduction of J mesons at HERA [22,23].

\section{Acknowledgements}

We thank S. Frixione, E. Laenen and M. Krämer for providing us with NLO QCD calculations for the process of interest and G.A. Schuler for the numerical integration program of the photon flux. We wish to express our gratitude to the CERN accelerator divisions for the excellent performance of the LEP machine. We also acknowledge and appreciate the effort of the engineers, technicians and support staff who have participated in the construction and maintenance of this experiment.

\section{References}

[1] M. Drees et al., Phys. Lett. B 306 (1993) 371.

[2] L3 Collaboration, B. Adeva et al., Nucl. Instrum. Methods A 289 (1990) 35;

L3 Collaboration, O. Adriani et al., Phys. Rep. 236 (1993) 1;
M. Acciarri et al., Nucl. Instrum. Methods A 351 (1994) 300; M. Chemarin et al., Nucl. Instrum. Methods A 349 (1994) 345; I.C. Brock et al., Nucl. Instrum. Methods A 381 (1996) 236; G. Basti et al., Nucl. Instrum. Methods A 374 (1996) 293; A. Adam et al., Nucl. Instrum. Methods A 383 (1996) 342.

[3] L3 Collaboration, M. Acciarri et al., Phys. Lett. B 453 (1999) 83.

[4] L3 Collaboration, M. Acciarri et al., Phys. Lett. B 503 (2001) 10.

[5] PYTHIA version 5.722 is used;

T. Sjöstrand, Preprint CERN-TH/7112/93 (1993), revised August 1995;

T. Sjöstrand, Comp. Phys. Commun. 82 (1994) 74.

[6] M. Cacciari et al., Nucl. Phys. B 466 (1996) 173.

[7] G.A. Schuler, T. Sjöstrand, Z. Phys. C 68 (1995) 607; G.A. Schuler, T. Sjöstrand, Phys. Lett. B 376 (1996) 193.

[8] V.M. Budnev et al., Phys. Rep. 15 (1975) 181.

[9] J.A.M. Vermaseren, Nucl. Phys. B 229 (1983) 347.

[10] KORALZ version 4.02 is used; S. Jadach, B.F.L. Ward, Z. Wạs, Comp. Phys. Commun. 79 (1994) 503.

[11] KORALW version 1.33 is used; S. Jadach et al., Comp. Phys. Commun. 94 (1996) 216; S. Jadach et al., Phys. Lett. B 372 (1996) 289.

[12] GEANT version 3.15 is used; R. Brun et al., Preprint CERN DD/EE/84-1 (1984), revised 1987.

[13] H. Fesefeldt, RWTH Aachen Report PITHA 85/2 (1985).

[14] G. D’Agostini, Nucl. Instrum. Methods A 362 (1995) 487.

[15] PHOJET version $1.05 \mathrm{c}$ is used; R. Engel, Z. Phys. C 66 (1995) 203; R. Engel, J. Ranft, Phys. Rev. D 54 (1996) 4246.

[16] D.E. Groom et al., Review of Particle Physics, Eur. Phys. J. C 15 (2000) 1.

[17] S. Frixione, E. Laenen, M. Krämer, Nucl. Phys. B 571 (2000) 169 ;

S. Frixione, E. Laenen, M. Krämer, J. Phys. G 26 (2000) 723.

[18] M. Gluck, E. Reya, I. Schienbein, Phys. Rev. D 60 (1999) 054019.

[19] L3 Collaboration, M. Acciarri et al., Phys. Lett. B 408 (1997) 450.

[20] A. Donnachie, P.V. Landshoff, Phys. Lett. B 296 (1992) 227.

[21] A. Donnachie, P.V. Landshoff, Phys. Lett. B 437 (1998) 408.

[22] H1 Collaboration, S. Aid et al., Nucl. Phys. B 472 (1996) 3.

[23] ZEUS Collaboration, G. Breitweg et al., Z. Phys. C 75 (1997) 215 . 